\title{
From Business Value Model to Coordination Process Model
}

\author{
Hassan Fatemi, Marten van Sinderen, and Roel Wieringa \\ Information Systems (IS) Research Group, \\ Electrical Engineering, Mathematics and Computer Science (EEMCS) Department, \\ University of Twente, Enschede, The Netherlands \\ h.fatemi@utwente.nl, m.j.vansinderen@ewi.utwente.nl, roelw@cs.utwente.nl
}

\begin{abstract}
The increased complexity of business webs calls for modeling the collaboration of enterprises from different perspectives, in particular the business and process perspectives, and for mutually aligning these perspectives. Business value modeling and coordination process modeling both are necessary for a good e-business design, but these activities have different goals and use different concepts. Nevertheless, the resulting models should be consistent with each other because they refer to the same system from different perspectives. Hence, checking the consistency between these models or producing one based on the other would be of high value. In this paper we discuss the issue of achieving consistency in multi-level e-business design and give guidelines to produce consistent coordination process models from business value models in a stepwise manner.
\end{abstract}

Keywords: Business value modeling, coordination process modeling, consistency checking, enterprise interoperability.

\section{Introduction}

Today, enterprises operate more and more together in networked collaborations rather than just on their own. There are many reasons for this. Among others we can refer to more complicated user needs, upward tendency toward specialization, changing customer demands, higher customer satisfaction indexes, etc. In the literature collections of enterprises that jointly satisfy a complex consumer need, are called business webs [1]. In a business web each enterprise contributes with its own specific expertise, products and services to satisfy a consumer need. For example, a web shop, logistics company, payment provider and authentication provider can jointly provide the service of on-line buying to consumers. Each partner wants to be sure that participation in such a collaboration network is economically profitable and sustainable before operational details of the coordination infrastructure are being designed. This is where business modeling comes into play. A coordination process model will be designed only if the business case is positive for each of the partners.

A coordination process model should specify the coordination activities (in terms of message exchanges) necessary to make the collaboration work. But, 
it is hard to design a coordination process model based on a business value model only, because there is a large conceptual gap between these two models 2. The main goal of business value modeling is to reach agreement amongst stakeholders regarding the question "Who is offering what of value to whom and expects what of value in return?" In contrast, an important goal of coordination process modeling is to reach a common understanding about which coordination activities should be carried out, and in which order. Business value modeling focuses on modeling economic sustainability of e-business while coordination process modeling focuses on modeling operational fulfillment. These are two different modeling goals, asking for different modeling methods with different constructs.

The research question addressed in this paper is: how to achieve consistency between business value models and coordination process models. The contribution of this paper consists of the description of initial guidelines to produce a coordination process model from a given business value model.

For representing the business value perspective, we use value models of $e^{3}$ value [3, and for the coordination process perspective, we use BPMN diagrams (see http://www.bpmn.org/). Our choice for $e^{3}$ value is motivated by the fact that it can express value transfers and value objects in general, as well as business actors. Our guidelines can be applied to any notation that can express this, but currently there is no other language that can express these things. The choice for BPMN is motivated by the fact that it is easy to read and well-known. Our guidelines can however be used with any other coordination language.

In section 2, we discuss the previous work published on this issue. Then, in section 3, we discuss about business value modeling and coordination process modeling and enumerate their similarities and differences. Based on these findings, we propose a stepwise approach to generate a coordination process model from a business value model in section 4 . In section 5 , we will apply our method on a case study and then analyze the results in section 6 . Finally we conclude with a discussion of results and future research in section 7 .

\section{Related Work}

The current approaches that consider the conceptual gap between business value models and coordination process models can be classified in two main groups:

- Consistency Checkers: These approaches assume that we have two models (a business value model and a coordination process model) of the same system and all we want is checking whether they are consistent with each other or not? Most of these approaches have solely considered consistency checking of static aspects, i.e., during design time and do not consider the runtime behavior of a model [45]6]. Bodenstaff [78] has introduced another approach toward consistency checking by considering the runtime behavior of the coordination process models. These approaches don't use the same definition for consistency and none of them is comprehensive enough to justify the consistency of these two models solely. 
- Model Generators: These approaches don't assume the availability of both a business value model and a coordination process model of the same system. Hence, they try to produce one from the other (mostly a coordination process model from a business value model) so that in the end both models be consistent with each other 91011 .

Pijpers and Gordijn proposed a method that makes an intermediate model ( $e^{3}$ transition model) based on the business value model by extending it with independent transfers of ownership rights of an object and the actual object itself.

Anderson and Bergholtz proposed a method that starts with a business value model and in a number of steps, each value exchange is analyzed and identified as a sub-process of the coordination process model. They break value exchanges to components (resource, right, custody, and document evidence).

Wieringa et al. claim that coordination modeling is facilitated by making a physical delivery model first, because the business and coordination model are both views of a network of physical deliveries. They distinguish discrete from cumulative goods and time continuous from time-discrete deliveries. They also specify frequency or duration of deliveries and make a delivery model as an intermediate model on the way to design a coordination process model.

In our opinion, these approaches are all too complicated because they ask for additional models and use complicated concepts such as ownership right, custody and physical delivery that makes it hard for others to use them in practice. Our proposed method is simpler, more general and comprehensive. By keeping it simple, we try to increase its applicability by other people.

\section{Business Value Models and Coordination Process Models}

As mentioned before, (business) value models and coordination (process) models are two models of the same system from different perspectives. A value model focuses on high level and static objects (e.g. value objects and actors) and value exchanges, while in contrast, a coordination model focuses on procedural details mainly message exchanges. A value model is built first because in that way participating companies can know as early as possible whether the cooperation will be economically sustainable for each of the partners. $e^{3}$ value is to our knowledge the only method that can do this with the level of formality that we need in order to be able to talk about consistency with coordination modeling.

In this section, we will pave the ground for the proposed method by enumerating the similarities and differences between value modeling and coordination modeling. Consider the $e^{3}$ value model (figure $1(\mathrm{a})$ ) and its corespondent coordination model in BPMN notation (figure 1(c)], in which a buyer a seller and a transporter exchange some values. The buyer gives money to the seller and receives good in return. The seller, in turn, gives money to the transporter and receives transport. We will discuss these models in detail later. 


\subsection{Differences}

The conceptual gap between value models and coordination models is caused mostly by the following properties of these models:

1. Ordering: The key concept in value modeling is value while its counterpart in coordination modelling is time. In an $e^{3}$ value model there is no notion of time ordering at all [3. The goal of a value model is to understand economic reciprocity of value transfers and to analyze the economic sustainability and profitability of the value activities of the actors. Behavior and temporal order are beyond the value perspective and are part of the coordination perspective. In addition, in value models there is no specific notion of cardinality or duration of value transfers and also it does not express preconditions of value transfers. But specifying preconditions of value transfers are an important part of business contracts.

2. Value versus coordination objects: In a value model every object should be of value to at least one partner (value object). Hence, there will be no place in a value model for those objects that are not of value to a partner. But in a coordination model objects are not included necessarily because they are of economic value to a partner. They can also be included because they help coordinating the activities of the partners. For example, messages are common objects that are used frequently in coordination models but, they don't have a particular economic value for any partner. Hence, they have no counterpart in value models. We call objects in the coordination model coordination objects.

3. Third parties: A direct value exchange between two partners in a value model does not necessarily imply that there will be a direct control object exchange between these partners in the corresponding coordination model. Sometimes a third party will be involved and the path for value object exchange becomes an indirect path for control object exchange. A well-known example for this case is the relation between buyer, seller and transporter in value model and coordination model. In this setting, there is a direct value exchange between the buyer and the seller, while the physical transfer of the good that is the subject of the value exchange will require an indirect control object exchange between the buyer and the seller involving a transporter. All three actors are present in a value model as well as in a coordination model, but the economic value interactions differ from the coordination interactions (see figure 1).

4. Paying methods: Money transfers are the most common transfers in value models that indicate paying a partner some money in exchange of his/her service or good. A money transfer between two partners in the value model, does not imply the paying method. There is a wide variety of payment methods for services and goods and this must be represented in the coordination model.

When moving from one type of model to the other, conceptual gap caused by the above four factors may have to be bridged. 


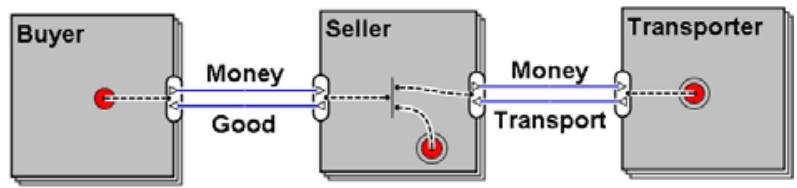

(a) Business Value model of Buyer, Seller and Transporter

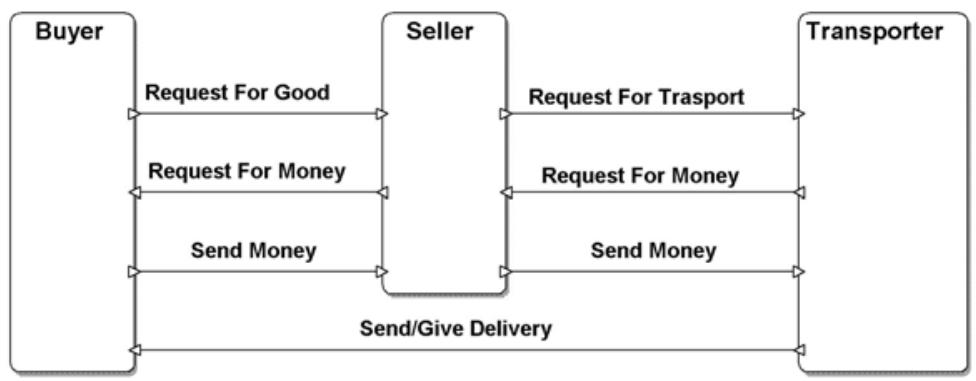

(b) All interactions (messages) Between Buyer, Seller and Transporter

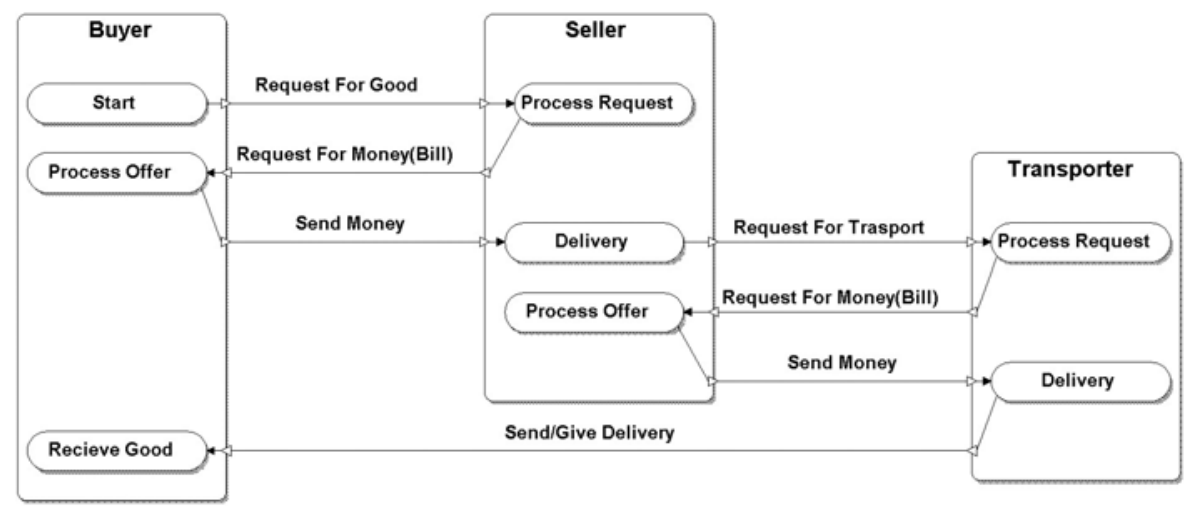

(c) Coordination Process model of Buyer, Seller and Transporter

Fig. 1. From Business Value model to Coordination Process model

\subsection{Similarities}

Despite the aforementioned conceptual gap, value modeling and coordination modeling also address some common aspects. First of all, they have the same actors/partners. In the business world, a partner joins a group only if (s)he earns something of value to herself/himself. Hence, every actor in a business network perceives some value and therefore will be present in the value model independently or as a part of another actor/partner. Because we are only interested in the way that independent actors cooperate with each other, rather than the 
internal organizational process, the coordination model contains only ordered interactions between independent actors.

Secondly, each value exchange, consisting of value transfers between two partners in different directions, indicates that something should happen to realize them. Different ways can be imagined to realize a value exchange. To start, we make some simplifying assumptions to reduce the complexity of the problem and converge different solutions.

In the coordination model we don't mention the activities that are internal to an actor, i.e. activities that don't involve communication with another actor. We don't take into consideration every necessary measure in message exchanges. For example when actor $A$ contacts actor $B$ three interactions can be imagined. First $A$ sends a request to $B$, then $B$ sends back a confirmation that it has received the request, and finally $A$ sends back $B$ the final confirmation. These interactions are necessary especially from the legal point of view in particular in situations that involve money transfers. But here for the sake of simplicity we have abstracted from them. This does not decrease the utility of our guidelines because any set of interactions between two actors can be preceded by a set of setup interactions without creating an inconsistency with the value model.

An important simplifying assumption is that all actors are trusted so that we don't need to consider security mechanisms to avoid mitigate the risk of frauds. In a realistic business model this assumption needs to be dropped but before building such a realistic model, the partners need to check whether the cooperation is economically sustainable (value model) and practically possible (coordination model) under the assumption that they can trust each other. If economic sustainability and practical possibility cannot be shown under the assumption of mutual trust, it is not worth the effort to check this under the more complicated conditions of lack of trust 12 . In this paper we therefore make this simplifying assumption but in future work we will drop it.

\section{From a Value Model to a Coordination Model}

The analysis above (section 3) will give a basis for the proposed method. The starting point is an $e^{3}$ value model. As an example, we consider the $e^{3}$ value model in figure $1(\mathrm{a})$.

Step 1: The first step is identifying the actors of the coordination model. As said in section 3.2 the actors in both value model and coordination model must be the same (buyer, seller, and transporter).

Step 2: In this step we aim at determining the necessary interactions that should be included in the coordination model to realize value exchanges. Under the simplifying assumptions mentioned in section 3.2 for each value transfer a pair of interactions (coordination objects) are enough to realize it. This pair consists of a request message and a message referring to the actual value object of the corresponding value transfer. Hence, we need four interactions for realizing each value exchange in the value model. In this way 
we cover the gap caused by the conceptual difference between value objects in the value model and coordination objects in the coordination model.

There is a special type of value exchange, which we call scheduled exchanges, that is excluded from this rule. An example of this type of value exchange is scheduled payment in which a partner pays an already determined amount of money for a service/good on already scheduled times. In these cases no party asks the other one for paying the money. Hence, in these cases in the coordination model we have only two interactions referring to the actual value objects.

Step 3: In this step we consider the third factor of the gap between value models and coordination models, namely third parties. For this purpose, we consider the following questions:

1. Who should send a request to whom? (Which partner to which other one?)

2. Who should give value to whom? (Which partner to which other one?)

In most of the cases, when there are no third parties in realizing value exchange, the answers to these questions are obvious and straightforward. In these cases, the partner that is going to receive the value object, sends a request message to the other one, that is going to send it, and then the latter sends the actual value to the requester. However, when third parties are involved in the process, we should exactly determine the sender and the receiver of the request message and the value object (See figure $1(\mathrm{~b})$. This model is an interaction model that shows who is causing the transfer of some observable object to whom.

At this point we see all the necessary interactions between partners, but still without any time ordering, so it is not yet a coordination model. Our next goal is to put these interactions in some ordering relationship and also determine the duration or cardinality of each of them.

Step 4: In order to put the interactions in a correct order in the coordination model we have to ask the following two questions regarding each value exchange of the value model:

\section{Who should first send a request to whom? (Which partner initiates?)}

2. Which value transfer should happen first?

Using the answers to these two questions we can put the four interactions in a correct order. Suppose that, for the case at hand, we have the following answers to the above two questions respectively:

1. The buyer should first send a request to the seller.

2. The seller gives the good to the buyer via the transporter.

The first answer is obvious and it indicates a request message from the buyer to the seller in the coordination model. However, the second answer indicates that the seller will not send the good to the buyer by himself/herself. Instead, 
(s)he should ask the transporter to do it. In other words, the seller should send a request message to the transporter to transport the good.

Step 5: After identifying the necessary interactions and putting them in the coordination model in a correct order, we can ask questions about time constraints to determine duration or cardinality characteristics of interactions. Using the answers to these questions, we can design the exact coordination model for the value model at hand. For example, suppose that $A$ provides service $S$ to $B$ for 1 year, and $B$ pays $A$ some money every month. In this case after including the necessary interactions in a correct order to the coordination model, we should add the duration property of the provisioning of the service and cardinality property of the payment.

Step 6: In this step we finalize the coordination model by adding the necessary and appropriate activities to each partner in order to link together the included interactions. In this way, starting from the partner with the start_stimulus (small circle), in the value model,we go forward in the model till we reach the partners with the end_stimulus (bull's eye). Now, the start and the stop activities can also be added to the model (See figure 1(c)) .

\section{Case Study}

To check whether our method indeed delivers a coordination model that is consistent its correspondent value model in a non-trivial case, we took an example that deals with the problem of clearing Intellectual Property Rights (IPR). It involves two steps: collecting fees from IPR users, i.e. radio stations, bars, discotheques and so on, and repartitioning the collected fees to Right Owners, i.e. artists, song writers, producers. The main IPR society interested in this problem is SENA (see http://www.sena.nl/).

Since 1993 SENA has been designated by the Ministry of Justice of The Netherlands to take care of the Neighboring Rights not only for Dutch right owners but also for foreign right owners of the music tracks in The Netherlands. It means whenever a track is played in public spaces with the aim of getting money from it, SENA must collect fees from such entities making money (IPR users) and repartition these fees to right owners (IPR owners). The aim is a payper-play scenario. It means that for each music track, a track-specific network of clearing organizations must be composed.

\subsection{Value Model}

The business model is based on the $e^{3}$ value methodology. The actors are:

Receivers: A receiver is an actor who broadcasts background music to get benefits of it so, they are also IPR users.

Background Music Providers (BMP): A BMP is an actor who provides specialized background music in exchange of fee. 


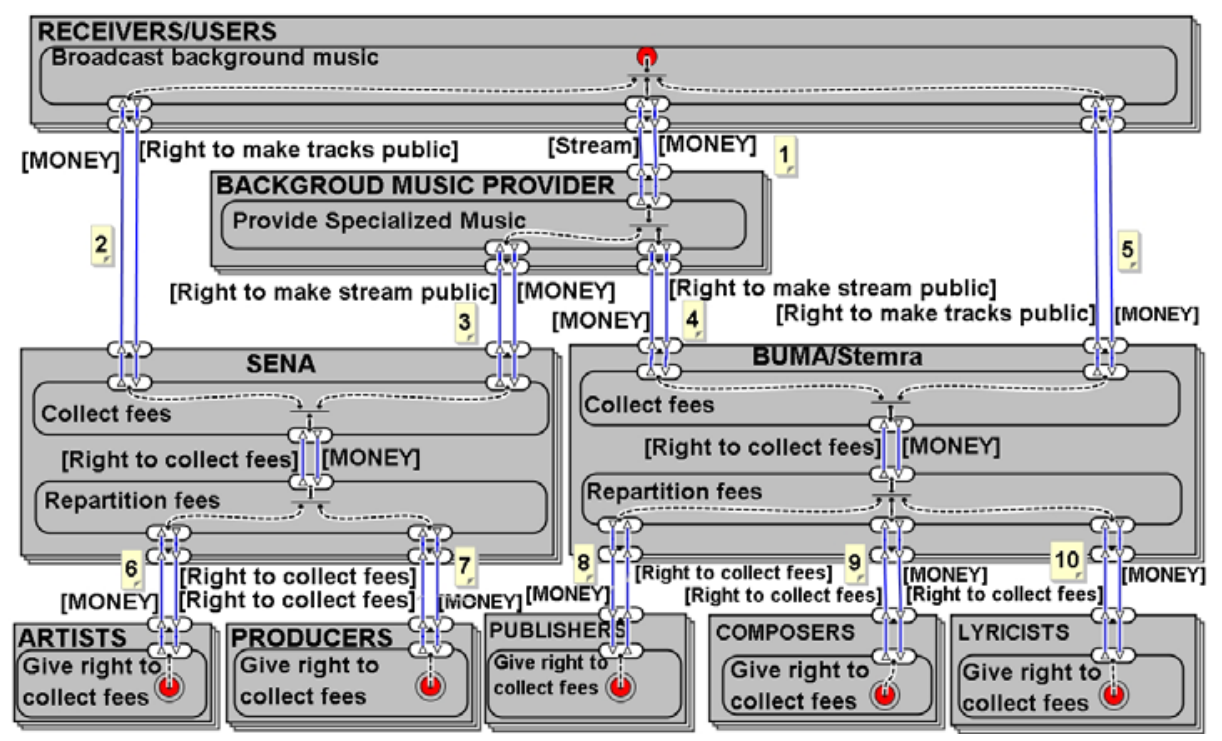

Fig. 2. Value model of providing music by Streaming

IPR Societies: IPR Societies perform mainly two roles; collecting fees for each played or copied track and repartitioning it to IPR owners. This fee is related with the public use of music (tracks). So, if an actor publicly provides music, it has to pay for the right of providing music.

Right Owners: Right owners are those who perform a specific track, i.e., play tracks, write lyrics, produce and publish tracks.

A BMP can provide background music in two ways. It can either deliver hard copies or a stream of tracks. Hard copies in this context are provided by a physical device in which a Receiver stores tracks provided by BMPs. On the other hand, a stream is a flow of tracks that the BMP delivers to a Receiver using Internetbased technology for direct playing. So, the main difference between these two ways of providing music is either allowing to store tracks at Receivers or not.

This main difference also generates two value models. If the BMP delivers hard copies, it must pay to IPR Societies which collect fees about replicating music, so making copies of tracks. Otherwise, when providing streams, the BMP must pay to IPR Societies which collect fees related with making a stream available to the public. Here we only investigate the second case (see figure 2).

To be able to provide music to the public, Receivers also have to pay IPR Societies. Paying BUMA/Stemra is about the copyright that the composer and/or lyricist holds, whereas paying SENA is related to the rights of the performing artists and producer. The process described so far is concerned with collecting fees. Therefore the next step is to repartition all those fees. SENA repartitions fees to Artists and Producers, and BUMA/Stemra does the same for Publishers, Composers and Lyricists. 


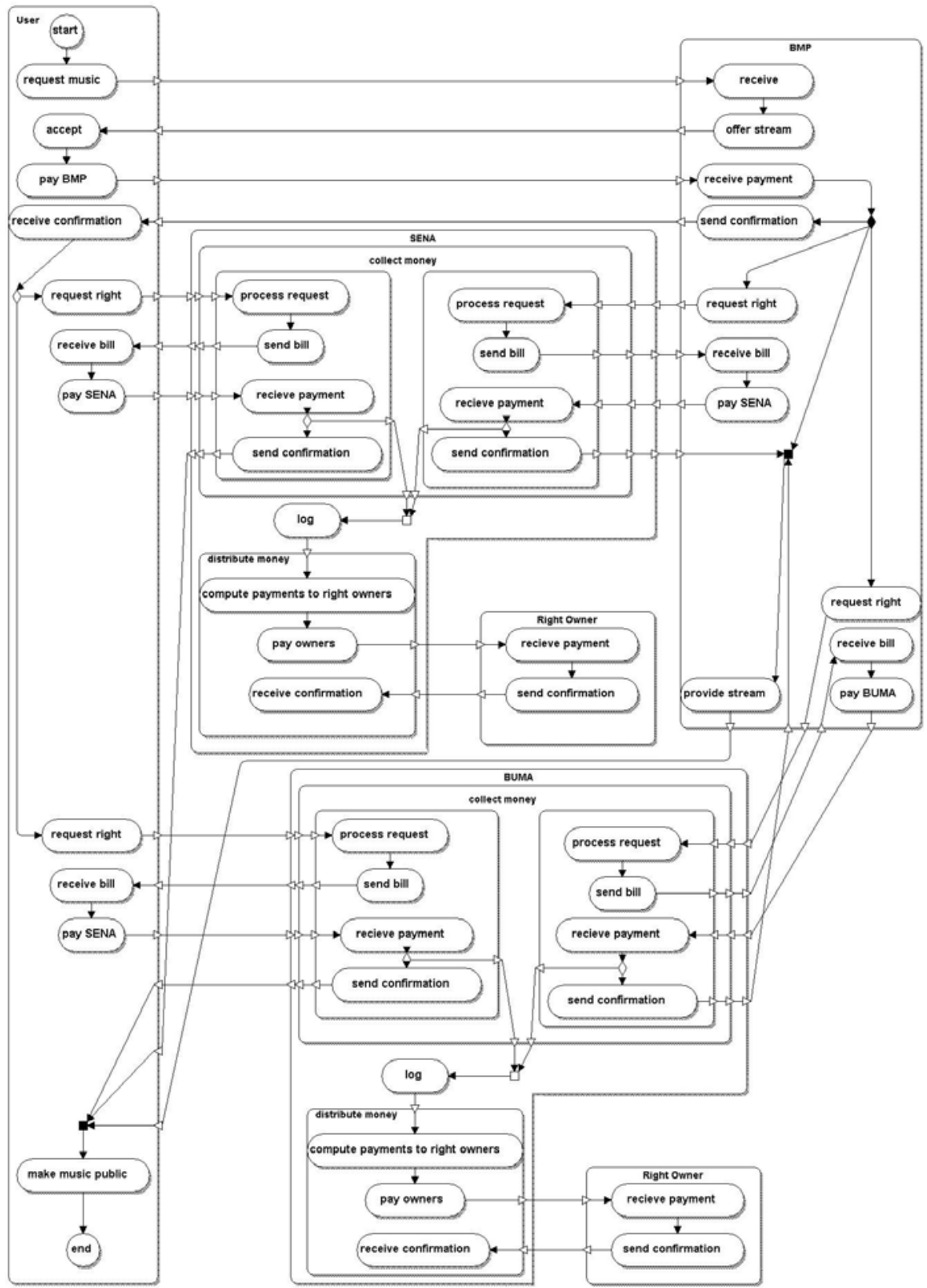

Fig. 3. Coordination model of providing music by Streaming 


\subsection{Coordination Model}

We applied our simple method to this case. The result is shown in figure 3 According to step 1 of the proposed method, actors are the same in the corresponding models. Because of the space limitation and the high similarity that is between right owners, we only include one right owner representing all of them. Therefore actors are: Receivers/Users, BMP, BUMA, SENA, and right owner.

In step 2, that is the most fundamental step, we identify the necessary interactions for realizing the value transfers. Under the simplifying assumptions we include four interactions to realize each pair of value transfers (a value exchange). The most obvious ones in this value model (figure 21) are those tagged as numbers $2,3,4$, and 5 . In all these four cases we have a pair of value transfers (money and right) being exchanged between two partners. Therefore according to step 2, using a pair of request-reply interactions they can be realized.

The value exchange tagged as number 1 , has a subtle difference with the proposed method. In this case the BMP sends back an extra confirmation to the Receiver/User. This extra confirmation is just for the sake of efficiency. If we remove this confirmation message, the Receiver/User has to wait until the arrival of the stream and after that do the necessary activities (payments), before being able to use the stream. The other value exchanges (numbers 6 through 10) are like scheduled payments described in step 2. Hence, we include only two interactions in to the coordination model to realize them.

We don't have the phenomenon of third parties thus we skip step 3. Step 4 is for putting the interactions in the correct order. Therefore, starting from the Receivers towards right owners we should find out the answers to the questions of this step regarding each value exchange and using the answers put the interactions in a correct order. Here we haven't consider the time constraints and durations because we suppose the provisioning of music as a simple service for which the duration is already determined. Also the way in which the payments are being done in real life depends on the situations and the agreements that have been made between payers and receivers. For example, one possible case is that BUMA and SENA pay the right owners in batch at the end of every month or so. But, here we consider only the simple case and leave considering all the details about payment methods and the cardinality or duration of the interactions to future work. In the last step we include the activities in the coordination model and using them connect the interactions to each other. We can also add details and other necessary activities. For example, for this particular case we could include the $\log$ as an additional activity in SENA and BUMA.

\section{Analysis of Results}

The proposed guidelines make a simple method that avoids complicated concepts like property right, physical delivery, etc. and still is able to guide the modeler to a coordination model that on face value is consistent with the value model. By applying our method on the above case study we learned that: 
- The application of these guidelines will indeed lead to a coordination model.

- The obtained coordination model is valid in the following sense: A stakeholder who accepts the value model ("this is how we will earn money in this business web") will also accept the coordination model ("this is how we will coordinate our actions with other actors in order to realize the value model").

We still want to do the following in order to further validate and improve our method:

- Comparison with the other methods. We observe that the papers do not give enough information to apply those methods to the described case (the bits of missing information concerning those methods will be identified in future work). But we will in the future apply our own method to more cases, and report the results.

- Apply our method to more complex cases to test its general applicability.

- Define an integrated consistency concept and prove that our method delivers consistent models.

\section{Conclusion and Future Work}

In this paper we have discussed the problem of how to go from a business/value model to a coordination coordination model in a stepwise and systematic way. Thanks to the conceptual commonalities that exist between the two models, a method could be proposed that starts with a value model where the main actors and their relationships, in the form of value exchanges, are identified. In a number of steps each value exchange is analyzed and by answering specific questions a coordination model is designed. The coordination model represents the interactions and interdependencies between the cooperating parties in terms of exchanged messages. We consider a special collection of interactions to realize the value exchanges of value models.

Future research involves eliminating different simplification assumptions such as trust that we have made here in constructing the coordination model. Another topic for future research includes investigations on different paying methods and finding a comprehensive collection of the most common ones to be able to make some patterns for paying methods and go from one to the other automatically.

\section{Acknowledgments}

The authors would like to thank the colleagues Anna Chmielowiec, Ivn S. RazoZapata, and Patricio de Alencar Silva in SENA case study. In particular, we thank Lianne Bodenstaff for her valuable contribution towards this work. 


\section{References}

1. Tapscott, D., Ticoll, D., Lowy, A.: Digital capital: harnessing the power of business webs. Ubiquity 1(13), 3 (2000)

2. Gordijn, J., Akkermans, H., van Vliet, H.: Business modelling is not process modelling. In: ER Workshops, pp. 40-51 (2000)

3. Gordijn, J., Akkermans, H.: Value Based Requirements Engineering: Exploring Innovative e-Commerce Ideas. Requirements Engineering Journal 8, 114-134 (2002)

4. Pijpers, V., Gordijn, J.: Consistency checking between value models and process models; a best-of-breed approach. Accepted at the BUSITAL (2008)

5. Zarvić, N., Wieringa, R., van Eck, P.: Checking the alignment of value-based business models and it functionality. In: SAC 2008: Proceedings of the 2008 ACM symposium on Applied computing, pp. 607-613. ACM, New York (2008)

6. Zlatev, Z., Wombacher, A.: Consistency between e3-value models and activity diagrams in a multi-perspective development method. In: Meersman, R., Tari, Z. (eds.) OTM 2005. LNCS, vol. 3760, pp. 520-538. Springer, Heidelberg (2005)

7. Bodenstaff, L., Wombacher, A., Reichert, M.U.: On formal consistency between value and coordination models. Technical Report TR-CTIT-07-91, Enschede (October 2007)

8. Bodenstaff, L., Wombacher, A., Reichert, M.U.: Dynamic consistency between value and coordination models - research issues. Technical Report TR-CTIT-06-50, Enschede (2006)

9. Pijpers, V., Gordijn, J.: Bridging business value models and process models in aviation value webs via possession rights. In: HICSS '07: Proceedings of the 40th Annual Hawaii International Conference on System Sciences, Washington, DC, USA, p. 175a. IEEE Computer Society, Los Alamitos (2007)

10. Andersson, B., Bergholtz, M., Grégoire, B., Johannesson, P., Schmitt, M., Zdvavkovic, J.: From business to process models-a chaining methodology. Conference paper (2006)

11. Wieringa, R., Pijpers, V., Bodenstaff, L., Gordijn, J.: Value-driven coordination process design using physical delivery models. In: Li, Q., Spaccapietra, S., Yu, E., Olivé, A. (eds.) ER 2008. LNCS, vol. 5231, pp. 216-231. Springer, Heidelberg (2008)

12. Wieringa, R., Gordijn, J.: Value-oriented design of correct service coordination processes: Correctness and trust. In: 20th ACM Symposium on Applied Computing, March 13-17, pp. 1320-1327. ACM Press, New York (2005) 